\title{
Hemodynamic analysis of radiation-induced damage in common carotid arteries by using color Doppler ultrasonography
}

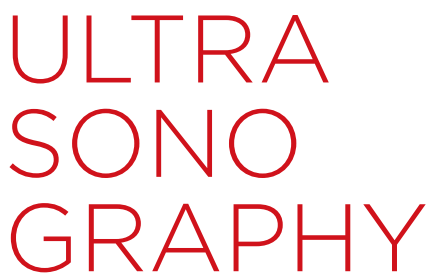

\author{
Alireza Mohammadkarim', Manijhe Mokhtari-Dizaji ${ }^{1}$, Ali Kazemian², Hazhir Saberi ${ }^{3}$ \\ 'Department of Medical Physics, Faculty of Medical Sciences, Tarbiat Modares University, \\ Tehran; ${ }^{2}$ Radiation Oncology Research Center, Cancer Institute, Tehran University of Medical \\ Sciences, Tehran; ${ }^{3}$ Department of Radiology, Imam Khomeini Hospital, Tehran University of \\ Medical Sciences, Tehran, Iran
}

\section{ORIGINAL ARTICLE}

https://doi.org/10.14366/usg. 17016 pISSN: 2288-5919 - eISSN: 2288-5943 Ultrasonography 2018;37:43-49

Received: February 17, 2017

Revised: April 14, 2017

Accepted: April 14, 2017

Correspondence to: Manijhe Mokhtari-Dizaji, PhD, Department of Medical Physics, Faculty of Medical Sciences, Tarbiat Modares University, Jalal AleAhmad Highway, Nasr Bridge, P.O. Box 1411713116, Tehran, Iran

Tel. $+98-21-82883893$

Fax. +98-21-88006544

E-mail: mokhtarm@modares.ac.ir

This is an Open Access article distributed under the terms of the Creative Commons Attribution NonCommercial License (http://creativecommons.org/ licenses/by-nc/3.0/) which permits unrestricted noncommercial use, distribution, and reproduction in any medium, provided the original work is properly cited.

Copyright @ 2018 Korean Society of Ultrasound in Medicine (KSUM)

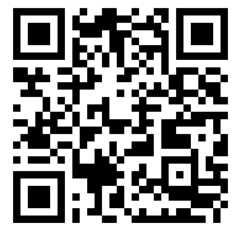

\section{Introduction}

External radiotherapy as a single modality or in combination with chemotherapy or surgery is a common treatment for tumors of the head and neck area [1-4]. The carotid arteries are the major vessels in the neck that supply blood to the brain, face, and neck. The main goal of external
How to cite this article:

Mohammadkarim A, Mokhtari-Dizaji M Kazemian A, Saberi H. Hemodynamic analysis of radiation-induced damage in common carotid arteries by using color Doppler ultrasonography. Ultrasonography. 2018 Jan;37(1):43-49. 
radiotherapy is to deliver the maximum dose to a tumor while achieving the minimum dose to the normal tissues. Despite technical advances in treatment planning systems, such as 3-dimensional conformal radiation therapy, intensity-modulated radiation therapy, and image-guided radiation therapy, it has not been possible to eliminate radiation exposure to the carotid arteries during radiotherapy in patients with head and neck carcinomas [5].

Post-irradiation damage to the carotid arteries is one of the most common undesirable effects of external radiotherapy in patients with malignancies who receive external irradiation to the head and neck region. In recent years, an increasing number of papers have been published reporting a link between external radiotherapy to the neck and the risk of stroke [5-7]. After irradiation, late injuries such as accelerated arteriosclerosis and carotid artery stenosis may occur in such patients. These vascular events lead to ischemic strokes and ischemic attacks in some cases as late adverse effects of radiotherapy $[5,7]$. Moreover, complications in the carotid arteries due to radiation, such as carotid blowout, pseudoaneurysm, and increased intima-media thickening have been reported in previous studies $[1,2,6-11]$. According to the findings of other studies $[2,12]$, the same dose of ionizing radiation has the same effects on the structural properties of the right and left common carotid arteries. Additionally, these complications play an important role in the assessment of the mechanical behavior of radiation-treated carotid arteries, but it seems that they have little sensitivity for the evaluation of the response of blood flow changes to followup radiotherapy procedures. It has previously been shown that investigating hemodynamic parameters is useful for the evaluation of vasodilation and stenosis in studies of vascular disorders $[13,14]$. Notably, the probability of blood supply abnormalities has rarely been described after external radiotherapy for the treatment of head and neck cancers.

Under clinical conditions, functional changes in the carotid arteries during procedures that affect the blood supply can be assumed to be correlated with the ultrasonic parameters of the common carotid arteries $[15,16]$. Noninvasive testing includes measuring the changes induced in blood dynamics with color Doppler imaging, which is a routine method of hemodynamic analysis. Additionally, automatic analysis for the calculation of instantaneous changes in vascular function using B-mode ultrasound images has been reported to be the most accurate and repeatable method for the diagnosis of blood vessel injuries [13].

To our knowledge, no study has comprehensively characterized hemodynamic parameters to evaluate the acute functional effects of neck-region radiotherapy on radiation-treated carotid arteries. In this study, the prevalence of abnormal carotid blood supply in patients exposed to head and neck external irradiation was examined by color Doppler measurements and by performing calculations with an image processing algorithm to determine quantitatively the changes in hemodynamic parameters in these subjects. Then, the relative deviation between the measured volume flow (VF) evaluated using the color Doppler system and the mean VF derived from the computational procedures was determined. Additionally, a statistical analysis of carotid VF parameter changes in the systolic and diastolic phases of the cardiac cycle was performed, and the acute influence of radiotherapy on the hemodynamic parameters of radiationtreated common carotid arteries was assessed.

\section{Materials and Methods}

\section{Study Population}

After approval by the Medical Ethics Committee of Tarbiat Modares University, this research was performed among patients who underwent carotid-region irradiation. Over a 7-month period, from August 2015 to March 2016, a total of 24 consecutive head and neck carcinoma patients who received primary or postoperative treatment at the Cancer Institute of Imam Khomeini Hospital in Tehran were studied before and after the external radiotherapy process. All the patients were treated by 6-MV photons and an isocentric technique delivering 2 Gy per day over 5 consecutive days per week (Varian Medical Systems, Palo Alto, CA, USA). The absorbed dose to the common carotid artery was extracted from the dose plan provided to the patient. Fifteen patients were men and nine patients were women. The participants in the study were adults, and none had a history of previous stroke or vascular surgery. The demographic and clinical characteristics of the study population are summarized in Table 1. It should be noted that informed consent for the examination was obtained from each patient in written form.

Because of differences in the tumors and treatment protocols among the subjects, unequal doses were delivered to the two carotid arteries. Hence, the present study was performed on the carotid artery that received a greater radiation dose during treatment based

Table 1. General characteristics of patients

\begin{tabular}{ll}
\hline \multicolumn{1}{c}{ Characteristic } & No. (\%) \\
\hline Age, mean \pm SD (yr) & $57 \pm 15$ \\
Male sex & $15(62.5)$ \\
Female sex & $9(37.5)$ \\
Vascular disease & $4(16.7)$ \\
High cholesterol & $4(16.7)$ \\
Hypertension & $3(12.5)$ \\
Tobacco use & $5(20.8)$ \\
Chemotherapy & $9(37.5)$ \\
\hline
\end{tabular}


on the location of the tumor. The tumor type and radiotherapy doses administered to subjects are shown in Table 2.

\section{Ultrasonography}

After at least 10 minutes of rest in the supine position, the ultrasonographic examination of the common carotid artery that received a greater radiation dose during treatment, $2-3 \mathrm{~cm}$ beneath the bifurcation region, was carried out as described in a previous study [17]. The SSI-6000 model of the Sonoscape ultrasonography system (SSI-6000 model, Shenzhen, China) equipped with a 6-11 $\mathrm{MHz}$ linear transducer was employed. Ultrasonic images were obtained at focal depth of $4.4 \mathrm{~cm}$. First, the cross-sectional diameter

Table 2. Cancer characteristics and radiation doses

\begin{tabular}{lll}
\hline \multicolumn{1}{c}{ Patient characteristics } & Patient & Dose (Gy) \\
\hline Tumor site & & \\
Oral cavity & $9(25.0)$ & $57 \pm 7$ \\
Larynx & $8(20.8)$ & $70 \pm 0$ \\
Parotid & $2(8.3)$ & $50 \pm 14$ \\
Lymphoma & $2(8.3)$ & $40 \pm 0$ \\
Oropharyngeal region & $1(4.2)$ & $70 \pm 0$ \\
Glomus & $1(4.2)$ & $50 \pm 0$ \\
Thyroid & $1(4.2)$ & $20 \pm 0$ \\
Mean dose of all subjects & & $59 \pm 11$ \\
\hline
\end{tabular}

Values are presented as number (\%) or mean \pm SD. of the common carotid artery was determined for each subject (Fig. 1). Then, blood velocity parameters were extracted from the color Doppler examination, including the peak systolic velocity $\left(V_{p}\right)$, end diastolic velocity $\left(V_{e}\right)$, and mean velocity $\left(V_{M}\right)$ of the common carotid artery. The following color Doppler parameters were calculated: the pulsatility index (PI), the resistive index (RI), and the systolic-todiastolic velocity (S/D) ratio. The arterial cross-section was extracted by B-mode images throughout three cardiac cycles, and the blood VF was reported for each subject. It is worth noting that the color Doppler settings were the same for all tests when the common carotid artery parameters were measured. While each test was performed, the probe was positioned at the lateral side of the neck on the carotid artery in the longitudinal view.

Instantaneous changes in the diameter of common carotid artery were evaluated using consecutive B-mode ultrasound images. For this purpose, sequential images of the common carotid arteries in the AVI format (65 frames per second) were transferred to a personal computer for processing. Sequential images were exported to MATLAB (ver. 7.10.0.499, Math Work Inc., Natick, MA, USA) for extraction of BMP images from the AVI movies. The peak systolic diameter $\left(d_{p}\right)$, the end diastolic diameter $\left(d_{e}\right)$, and the mean-value diameter $\left(d_{M}\right)$ of each lumen were determined using the maximum gradient algorithm in MATLAB, which allowed the quantitative assessment of the time-dependent movements of the vascular wall from the sequential ultrasonic images obtained throughout three cardiac cycles (Fig. 2). The essential features of this algorithm have been described in detail elsewhere [18].

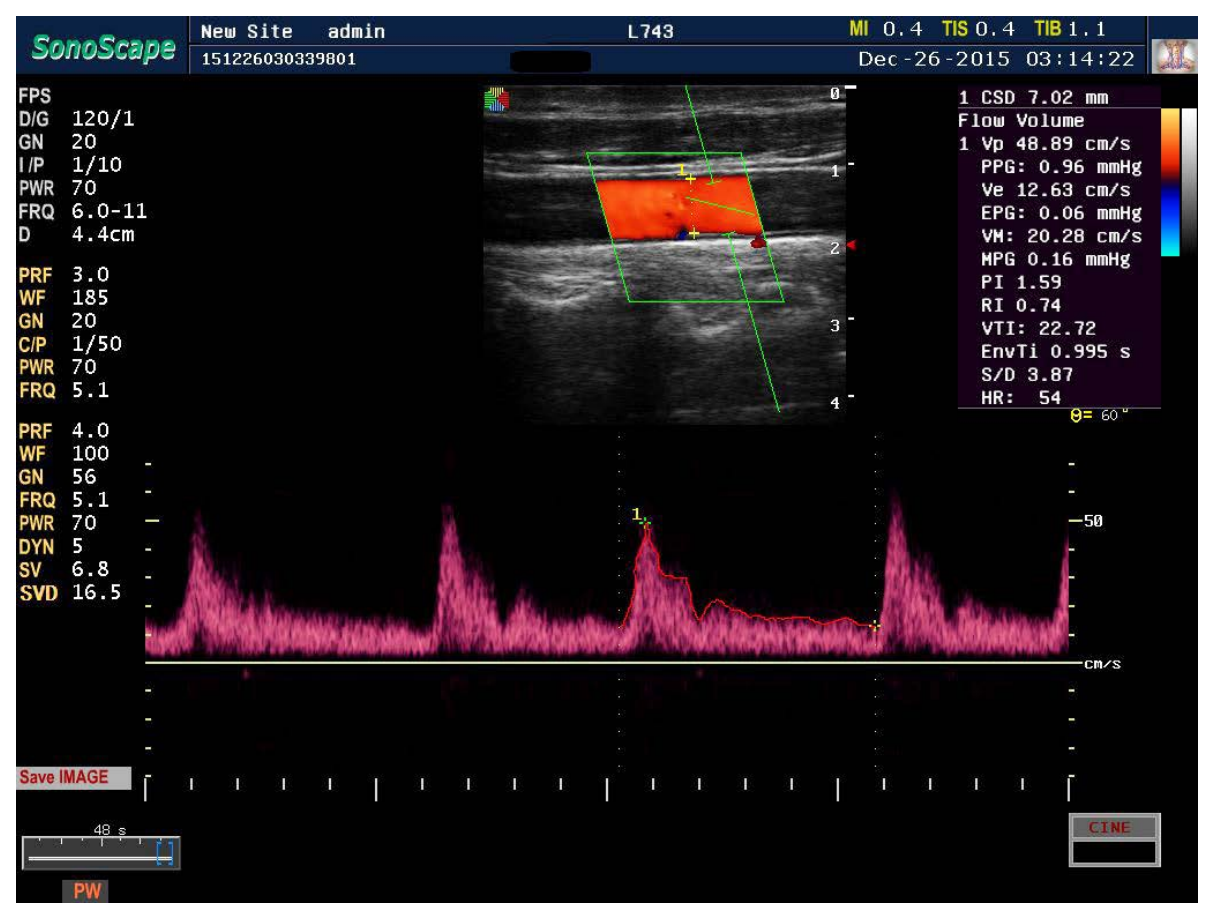

Fig. 1. Color Doppler ultrasonography of the common carotid artery with a blood velocity spectrum. The diameter of the common carotid artery was determined for each subject. The peak systolic velocity $\left(V_{p}\right)$, end diastolic velocity $\left(V_{e}\right)$, and mean velocity $\left(V_{M}\right)$ of the common carotid artery were extracted from the color Doppler examination. 


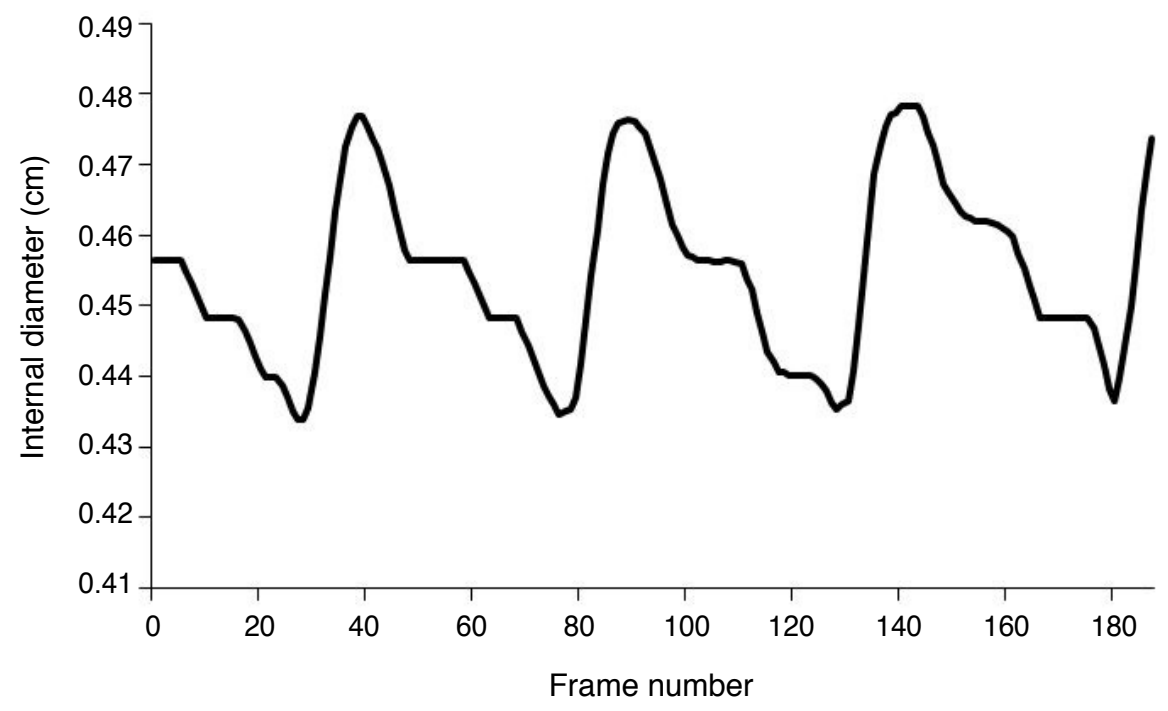

Fig. 2. Instantaneous changes in the internal diameter of the common carotid artery throughout three cardiac cycles, evaluated using the computational method. The peak systolic diameter $\left(d_{p}\right)$, end diastolic diameter $\left(d_{e}\right)$, and mean value diameter $\left(d_{M}\right)$ of each lumen were determined from sequential ultrasonic images throughout three cardiac cycles.
Blood $V F$ at the peak systolic mode $\left(V F_{p}\right)$, the end diastolic mode $\left(V F_{e}\right)$, and the mean value of $V F\left(V F_{M}\right)$ were extracted using the following formulas.

$$
\begin{aligned}
& V F_{p}=\pi\left(\frac{d_{p}}{2}\right)^{2} \times V_{p} \\
& V F_{e}=\pi\left(\frac{d_{e}}{2}\right)^{2} \times V_{e} \\
& V F_{M}=\pi\left(\frac{d_{M}}{2}\right)^{2} \times V_{M}
\end{aligned}
$$

For assessment of the early effects of radiotherapy, color Doppler ultrasonography and B-mode ultrasonic images were acquired for each subject before radiotherapy was begun and after the last session of fraction dose delivery, as reported by Adriaenssens et al. [19].

Finally, the relative difference $(\Delta)$ of the two methods of assessing VF was evaluated by comparing the calculated VF derived from the computational method $\left(\mathrm{VF}_{\text {cal }}\right)$ and the routine measurement of blood $V F\left(V F_{\text {meas }}\right)$, as seen in the following equation.

$$
\Delta=\frac{V F_{\text {cal }}-V F_{\text {meas }}}{V F_{\text {meas }}} \times 100
$$

\section{Statistical Analysis}

In the present study, the necessary sample size was estimated to be 10 with a confidence level of $95 \%$ and $90 \%$ power. Therefore, 24 patients (mean age, 57 years) were enrolled in this study. All subjects underwent color Doppler ultrasonography. The hemodynamic data of each patient before and after treatment that were derived from the Doppler ultrasonography system and the mathematical procedure based on the detection of instantaneous motion in the common carotid arterial walls were analyzed using the paired-sample $t$ test to compare the baseline hemodynamic parameters with probable abnormalities in the ultrasonic Doppler parameters of blood flow following treatment. $\mathrm{P}<0.05$ were considered to indicate statistical significance.

\section{Results}

The color Doppler parameters related to blood flow velocity that were used to evaluate the acute effects of ionizing irradiation are listed in Table 3. The treated patients had significantly more lumen constriction than the baseline group based on the internal diameter measured using the Doppler ultrasonography system and the detection of instantaneous motion in the common carotid arterial wall (Table 3).

A significant increase was found in the mean velocity, $\mathrm{S} / \mathrm{D}$ ratio, $\mathrm{PI}$, and $\mathrm{Rl}$ after external radiotherapy. Radiotherapy treatment in head and neck cancer patients especially significantly reduced the internal diameter and blood VF of the common carotid arteries. Additionally, the RI parameter increased more significantly than other measured parameters derived from the blood flow velocity profile. Among these parameters, no significant changes were seen in the peak systolic velocity between the baseline group and the post-treatment group $(P=0.120)$. The treated patients had significantly greater lumen constriction than the baseline group based on the internal 
Table 3. Lumen diameter and blood flow velocity parameters of the common carotid arteries before and after external radiotherapy

\begin{tabular}{lccr}
\hline \multicolumn{1}{c}{ Parameter } & Pre-irradiation & Post-irradiation & P-value \\
\hline $\begin{array}{l}\text { Peak systolic velocity } \\
\text { (cm/sec) }\end{array}$ & $47.80 \pm 9.72$ & $55.26 \pm 13.24$ & 0.120 \\
$\begin{array}{l}\text { End diastolic velocity } \\
\text { (cm/sec) }\end{array}$ & $12.83 \pm 2.68$ & $11.09 \pm 3.64$ & 0.025 \\
Mean velocity (cm/sec) & $22.88 \pm 4.57$ & $23.3 \pm 5.18$ & 0.035 \\
Pulsatility index & $1.58 \pm 0.37$ & $1.78 \pm 0.43$ & 0.027 \\
Resistive index & $0.73 \pm 0.06$ & $0.79 \pm 0.05$ & $<0.001$ \\
Systolic to diastolic & $4.04 \pm 0.98$ & $4.88 \pm 1.31$ & 0.010 \\
velocity ratio & & & \\
$\mathrm{d}_{\text {meas }}(\mathrm{mm})$ & $6.12 \pm 0.54$ & $5.26 \pm 0.52$ & $<0.001$ \\
$\mathrm{~d}_{\mathrm{p}}(\mathrm{mm})$ & $6.42 \pm 0.67$ & $5.63 \pm 0.67$ & $<0.001$ \\
$\mathrm{~d}_{\mathrm{e}}(\mathrm{mm})$ & $5.56 \pm 0.58$ & $4.92 \pm 0.55$ & $<0.001$ \\
$\mathrm{~d}_{\mathrm{M}}(\mathrm{mm})$ & $6.05 \pm 0.64$ & $5.29 \pm 0.60$ & $<0.001$ \\
\hline
\end{tabular}

Lumen diameter parameters were obtained by two methods: a clinical investigation $\left(\mathrm{d}_{\text {meas }}\right)$ and the automated diameter that was calculated using the image processing procedure $\left(d_{p}, d_{e}, d_{M}\right)$.

diameter measured using the Doppler ultrasonography system and the detection of instantaneous motion in the common carotid arterial wall (Table 3).

Therefore, the blood VF values of radiated carotid arteries were lower than the non-radiated arteries $(P<0.05)$.

Table 4 shows the mean \pm standard deviation and $P$-values of measured and estimated blood VF. Blood VF was significantly lower in all subjects following external irradiation than at baseline. A significant difference was found between the blood flow volume in the peak systolic phase and the end diastolic phase, as well as the mean blood flow volume of the common carotid arteries after radiotherapy compared to before treatment $(P<0.05)$.

The relative difference between the measured $\mathrm{VF}_{\text {meas }}$ which was used as the mean VF in the clinical investigation, and the automated $\mathrm{VF}_{\mathrm{M}}$, which was calculated using the image processing procedure, was found to be $10.63 \%$ and $10.01 \%$ in the pretreatment and radiation-treated groups, respectively. These results show that the routine measurements of VF may be accompanied by errors. However, the blood VF obtained from the clinical investigation was overestimated by $2 \%$ relative to the blood VF calculated based on the arterial diameter in the image processing method.

\section{Discussion}

It has been reported in qualitative studies that primary radiation to the neck increases the incidence of carotid arterial stenosis and may induce various types of structural damage $[15,20]$. The etiology
Table 4. Blood VF before and after external radiotherapy obtained using two methods: clinical investigations $\left(\mathrm{VF}_{\text {meas }}\right)$ and calculated using the image processing $\left(\mathrm{VF}_{\mathrm{p}}, \mathrm{VF}_{\mathrm{e}}\right.$, and $\left.\mathrm{VF}_{\mathrm{M}}\right)$

\begin{tabular}{|c|c|c|c|}
\hline Ultrasonic parameter & Pre-irradiation & Post-irradiation & P-value \\
\hline Blood VF $F_{\text {meas }}(\mathrm{mL} / \mathrm{min})$ & $395.20 \pm 36.39$ & $313.79 \pm 101.10$ & $<0.001$ \\
\hline Blood $V F_{p}(m L / m i n)$ & $926.18 \pm 218.42$ & $778.16 \pm 266.20$ & 0.003 \\
\hline Blood VF $(\mathrm{mL} / \mathrm{min})$ & $187.62 \pm 52.61$ & $125.63 \pm 46.11$ & $<0.001$ \\
\hline Blood VF $F_{M}(\mathrm{~mL} / \mathrm{min})$ & $387.94 \pm 105.80$ & $306.39 \pm 85.48$ & $<0.001$ \\
\hline
\end{tabular}

$\mathrm{VF}$, volume flow.

of radiation-induced carotid artery injury in the endothelium, media, and adventitia were discussed by Gujral et al. [15]. It was established in that study that early radiation damage is characterized by an inflammatory process focused on initial changes in the endothelial cells, followed by fibrosis and necrosis in the media and adventitia. However, apart from stenosis, functional injuries or changes, especially regarding the role of blood supply, in the carotid artery following external radiotherapy to the neck area have not yet been reported. In the present study, changes in the blood flow parameters of the carotid artery before and after irradiation indicated the presence of underlying vascular complications [15].

The Doppler ultrasonography technique showed significant increases in the mean values of the $\mathrm{Pl}$ and $\mathrm{RI}$ after radiotherapy (Table 3). Moreover, as shown in Table 3, significantly higher mean $S / D$ ratio values were obtained after radiation treatment than at baseline. The S/D ratio can properly predict unilateral and differential common carotid artery stenosis. As described in previous research, a markedly increased S/D ratio is a reliable marker of proximal severe stenosis [16]. It seems that the relatively large standard deviation values of the blood flow velocity parameters are due to the differences between various patients' arteries in terms of their primary health (Table 3). Of the subjects who underwent radiotherapy in the neck area, $75 \%$ showed some degree of carotid artery stenosis [12]. Other researchers have reported that $40 \%$ of radiation-treated carotid arteries showed significant carotid stenosis ( $\geq 60 \%$ stenosis), with $15 \%$ of subjects showing unilateral carotid occlusion, $15 \%$ of patients showing significant bilateral carotid stenosis, and $7.5 \%$ of patients having sustained a stroke after treatment with ionizing irradiation [20]. In the present research, highly significant changes in the common carotid artery diameter were also found in all the treated patients at the time of follow-up in comparison with the preradiotherapy values assessed using US system measurements and the computational method throughout three cardiac cycles (Table 4). This condition can be referred to as radiation-induced atherosclerotic carotid arteries [9]. In a recent report, arterial stiffness was introduced as a biomarker of radiation- 
induced carotid atherosclerosis [13]. An increase in the S/D ratio was seen in 23 patients (95.83\%) who underwent external radiotherapy in the head and neck region, and it is known to be an acute effect of ionizing radiation that is experienced as soon as the radiotherapy process is completed.

The quantification of blood VF is clinically useful because it allows the hemodynamic assessment of carotid artery stenosis and its collateral pathways and associated conditions [14,21]. With regard to changes in the blood VF parameters of patients (Table 4), non-negligible variations in VF occurred in the irradiated arteries compared with the baseline samples throughout the cardiac cycle. Since the blood VF is approximated by multiplying the luminal crosssectional area by the corresponding mean velocity of blood flow, and the luminal cross-section is proportional to $\mathrm{d}^{2} / 4$, a considerable decrease of blood VF occurred in the radiation-treated vessels. Although the variation within the mean velocity after radiotherapy was statistically significant, the numerical variation of this parameter was negligible (Table 3).

The effects of irradiation on color Doppler parameters of the inferior thyroid artery have previously been evaluated in order to assess functional changes in the thyroid gland. Significant increases in the $\mathrm{Pl}, \mathrm{RI}$, blood $\mathrm{VF}$, and the internal diameter of the lumen were observed after the completion of external radiotherapy in comparison with the values observed before the start of irradiation [22]. It should be noted that the mechanical structure of the inferior thyroid artery is different from that of the common carotid arteries, and it seems that the differences in the behavior of hemodynamic parameters after radiotherapy are due to such structural variations.

In the present study, we observed a larger common carotid diameter and higher blood VF values in males than in females, and in old subjects and patients with a history of smoking than in young subjects and nonsmoking patients, for both the irradiated and nonirradiated carotid arteries. However, we did not observe a significant difference between the behavior of $V F$ in the nonirradiated and irradiated carotid arteries according to age, gender, or history of smoking. Moreover, external radiation had the same influence on the hemodynamic parameters of carotid arteries with a normal intima-media dimension and carotid arteries with greater intima-media thickness. Moreover, a relatively uniform decrease in the blood VF was seen in the systolic and diastolic phases of the cardiac cycle. In addition, a significant decrease in the VF was observed using measurement-based and computational methods (Table 5). However, our results also illustrated that the $V F$ values derived from the ultrasonography system exhibited nonnegligible error.

In some previous studies $[1,7,8,10,20]$, carotid stenosis was reported in a follow-up test conducted at least 1 year after the patient's last radiation treatment as a late form of toxicity. In those studies, only the internal diameter of the radiation-induced vessels was evaluated, and blood supply parameters were ignored. In this study, a decrease was found in the diameter of the carotid artery in head and neck cancer patients after treatment with radiation. These results demonstrate the near-term effects of radiation-induced toxicity due to head and neck irradiation. In light of the findings of the present study, we suggest the immediate implementation of Doppler ultrasound imaging after the treatment process, especially for patients with atherosclerotic disease, based on Doppler imaging of the carotid artery prior to undergoing irradiation in order to promptly diagnose the early effects of radiation on the blood supply.

Although the present study demonstrated that hemodynamic parameters responded to radiotherapy, it has two main limitations. First, the dose delivery methodology during the treatment process was not exactly the same in all subjects, since the patients suffered from different types of head and neck tumors. Second, the longterm effects of radiotherapy on hemodynamic parameters were not investigated in this study.

In conclusion, external radiotherapy involving the head and neck region generally increases long-term survival, but may lead to carotid stenosis and failure of the blood supply in the short-term after cancer treatment. The feasibility of using Doppler ultrasonography and B-mode image processing for the objective assessment of carotid artery hemodynamics was investigated. The most interesting finding in this study was the identification of significant differences in hemodynamic parameters, especially blood VF, between baseline and post-treatment arteries throughout three cardiac cycles using an automatic computational procedure. In conclusion, it is clear that radiation-induced damage to the carotid artery can be regarded as a form of primary damage to the vessel function.

ORCID: Manijhe Mokhtari-Dizaji: http://orcid.org/0000-0003-1598-2038

\section{Conflict of Interest}

No potential conflict of interest relevant to this article was reported.

\section{Acknowledgments}

This study was approved by the Faculty of Medical Sciences, Tarbiat Modares University. This work was supported in part by the Iran National Science Foundation (INSF).

\section{References}

1. Carmody BJ, Arora S, Avena R, Curry KM, Simpkins J, Cosby K, et al. Accelerated carotid artery disease after high-dose head and neck radiotherapy: is there a role for routine carotid duplex surveillance? 
J Vasc Surg 1999;30:1045-1051.

2. Gianicolo ME, Gianicolo EA, Tramacere F, Andreassi MG, Portaluri M. Effects of external irradiation of the neck region on intima media thickness of the common carotid artery. Cardiovasc Ultrasound 2010;8:8.

3. Zheng YP, Leung SF, Mak AF. Assessment of neck tissue fibrosis using an ultrasound palpation system: a feasibility study. Med Biol Eng Comput 2000;38:497-502.

4. Maruyama Y, Arai K, Hoshida S, Yoneda K, Furukawa M, Yoshizaki T. Case of three delayed complications of radiotherapy: bilateral vocal cord immobility, esophageal obstruction and ruptured pseudoaneurysm of carotid artery. Auris Nasus Larynx 2009;36:505-508.

5. Garcez K, Lim CC, Whitehurst P, Thomson D, Ho KF, Lowe M, et al. Carotid dosimetry for $\mathrm{T} 1$ glottic cancer radiotherapy. Br J Radiol 2014;87:20130754.

6. Ellens DJ, Hurley MC, Surdel D, Shaibani A, Pelzer H, Bendok BR. Radiotherapy-induced common carotid pseudoaneurysm presenting with initially occult upper airway hemorrhage and successfully treated by endovascular stent graft. Am J Otolaryngol 2010;31:195198.

7. Chang YJ, Chang TC, Lee TH, Ryu SJ. Predictors of carotid artery stenosis after radiotherapy for head and neck cancers. J Vasc Surg 2009;50:280-285.

8. Brown PD, Foote RL, McLaughlin MP, Halyard MY, Ballman KV, Collie AC, et al. A historical prospective cohort study of carotid artery stenosis after radiotherapy for head and neck malignancies. Int J Radiat Oncol Biol Phys 2005;63:1361-1367.

9. Cheng SW, Ting AC, Wu LL. Ultrasonic analysis of plaque characteristics and intimal-medial thickness in radiationinduced atherosclerotic carotid arteries. Eur J Vasc Endovasc Surg 2002;24:499-504.

10. Martin JD, Buckley AR, Graeb D, Walman B, Salvian A, Hay JH. Carotid artery stenosis in asymptomatic patients who have received unilateral head-and-neck irradiation. Int J Radiat Oncol Biol Phys 2005;63:1197-1205.

11. McDonald MW, Moore MG, Johnstone PA. Risk of carotid blowout after reirradiation of the head and neck: a systematic review. Int J Radiat Oncol Biol Phys 2012;82:1083-1089.

12. Vitolo V, Millender LE, Quivey JM, Yom SS, Schechter NR, JereczekFossa BA, et al. Assessment of carotid artery dose in the treatment of nasopharyngeal cancer with IMRT versus conventional radiotherapy. Radiother Oncol 2009;90:213-220.

13. Gujral DM, Shah BN, Chahal NS, Bhattacharyya S, Senior R, Harrington KJ, et al. Arterial stiffness as a biomarker of radiationinduced carotid atherosclerosis. Angiology 2016;67:266-271.

14. Ho SS, Metreweli C. Preferred technique for blood flow volume measurement in cerebrovascular disease. Stroke 2000;31:13421345.

15. Gujral DM, Shah BN, Chahal NS, Senior R, Harrington KJ, Nutting CM. Clinical features of radiation-induced carotid atherosclerosis. Clin Oncol (R Coll Radiol) 2014;26:94-102.

16. Pisimisis GT, Katsavelis D, Mandviwala T, Barshes NR, Kougias P. Common carotid artery peak systolic velocity ratio predicts highgrade common carotid stenosis. J Vasc Surg 2015;62:951-957.

17. Soleimani E, Mokhtari-Dizaji M, Saberi H. A novel non-invasive ultrasonic method to assess total axial stress of the common carotid artery wall in healthy and atherosclerotic men. J Biomech 2015;48:1860-1867.

18. Rahmani-Cherati T, Mokhtari-Dizaji M, Vajhi A, Rostami A. Evaluation of statin therapy on endothelial function in hypercholesterolemic rabbits by automatic measurement of arterial wall movement using ultrasound images. Ultrasound Med Biol 2014;40:2415-2424.

19. Adriaenssens N, Belsack D, Buyl R, Ruggiero L, Breucq C, De Mey J, et al. Ultrasound elastography as an objective diagnostic measurement tool for lymphoedema of the treated breast in breast cancer patients following breast conserving surgery and radiotherapy. Radiol Oncol 2012;46:284-295.

20. Steele SR, Martin MJ, Mullenix PS, Crawford JV, Cuadrado DS, Andersen CA. Focused high-risk population screening for carotid arterial stenosis after radiation therapy for head and neck cancer. Am J Surg 2004;187:594-598.

21. Oktar SO, Yucel C, Karaosmanoglu D, Akkan K, Ozdemir H, Tokgoz $\mathrm{N}$, et al. Blood-flow volume quantification in internal carotid and vertebral arteries: comparison of 3 different ultrasound techniques with phase-contrast MR imaging. AJNR Am J Neuroradiol 2006;27:363-369.

22. Bakhshandeh M, Hashemi B, Mahdavi SR, Nikoofar A, Edraki HR, Kazemnejad A. Evaluation of thyroid disorders during head-andneck radiotherapy by using functional analysis and ultrasonography. Int J Radiat Oncol Biol Phys 2012;83:198-203. 